\title{
Existence and Uniqueness of a Minimum Crisp Boolean Petri Net
}

\author{
Gajendra Pratap Singh \\ Department of Applied Mathematics \\ Delhi Technological University \\ Shahbad Daulatpur, Main Bawana Road \\ Delhi-110042, India
}

\author{
Sangita Kansal \\ Department of Applied Mathematics \\ Delhi Technological University \\ Shahbad Daulatpur, Main Bawana Road \\ Delhi-110042, India
}

\author{
Mukti Acharya \\ Department of Applied Mathematics \\ Delhi Technological University \\ Shahbad Daulatpur, Main Bawana Road \\ Delhi-110042, India
}

\begin{abstract}
In the continuing research towards characterizing 1-safe Petri nets with $n$-places and generating all the $2^{n}$ binary $n$-vectors as marking vectors exactly once, the problem of determine minimum Petri nets; 'minimum' in the sense that the number of transitions is kept minimum possible for the generation of all the $2^{n}$ binary $n$-vectors has been found. In this paper, the existence and uniqueness of a minimum Petri net which generates all the $2^{n}$ binary $n$-vectors exactly once has been shown. For brevity, a 1-safe Petri net that generate all the binary $n$-vectors as marking vectors is called a Boolean Petri net and a 1-safe Petri net that generates all the binary $n$-vectors exactly once is called crisp Boolean Petri net.
\end{abstract}

\section{Keywords:}

1 -safe Petri net, reachability tree, binary $n$-vector, marking vector.

\section{INTRODUCTION}

Petri nets invented by C.A. Petri [1] are designed specifically to model a variety of systems, especially concurrent and dynamic systems, of which computer systems are a good representation. Petri nets can capture the essence of decisions: conflict and parallelism. Petri nets are the graphical and mathematical modeling tool applicable to many systems such as modeling, analysis, control, optimization, simulation and implementation of various engineering systems. Of all existing models, Petri nets and their extensions are of undeniable fundamental interest because they define easy graphical support for the representation and the understanding of basic mechanism and behaviors. The development of high-end computers has greatly enhanced the use of Petri nets in diverse fields. However, there is a drawback inherent to discrete event-system they suffer from state explosion problem as what will happen when a system is highly populated, i.e., initial marking is large. This phenomenon leads to an exponential growth of the cardinality of the set of markings which, in turn, would blow up the 'size' (i.e., the number of arcs) of the system. This makes us to study the safe systems deeply. Towards this end, the authors proposed a 1-safe star Petri net $S_{n}$, with $|P|=n$ and $|T|=n+1$, having a central transition, that generates all the binary $n$-vectors, as its marking vectors |2|; they also established the existence of 1-safe Petri nets that generate all the binary $n$-vectors exactly once as marking vectors keeping the depth of the reachability tree minimum [3]. After that a question came into the mind of authors that, "Does there exist a Petri net which generates all the binary $n$-vectors exactly once with minimum number of transitions?" and they successfully established the existence of such a Petri net in this paper. The uniqueness of this Petri net is also shown here.

\section{PRELIMINARIES}

For the sake of completeness, some of the necessary definitions and concepts used in this paper are discussed here. For standard terminology and notation on Petri nets theory and Graph theory, the reader is referred to Peterson[4] and Harary [5], respectively. Throughout this paper, the following definition given by Jensen [6] is being used.

A Petri net is a 5 -tuple $N=\left(P, T, I^{-}, I^{+}, \mu^{0}\right)$, where

(1) $P$ is a nonempty set of 'places',

(2) $T$ is a nonempty set of 'transitions',

(3) $P \cap T=\emptyset$,

(4) $I^{-}, I^{+}: P \times T \longrightarrow \mathbb{N}$, where $\mathbb{N}$ is the set of nonnegative integers, are called the negative and the positive 'incidence functions' (or, 'flow functions') respectively,

(5) $\forall p \in P, \exists t \in T: I^{-}(p, t) \neq 0$ or $I^{+}(p, t) \neq 0$ and $\forall t \in T, \exists p \in P: I^{-}(p, t) \neq 0$ or $I^{+}(p, t) \neq 0$,

(6) $\mu^{0}: P \rightarrow \mathbb{N}$ is the initial marking.

In fact, $I^{-}(p, t)$ and $I^{+}(p, t)$ represent the number of arcs from $p$ to $t$ and $t$ to $p$ respectively. $I^{-}, I^{+}$and $\mu^{0}$ can be viewed as matrices of size $|P| \times|T|,|P| \times|T|$ and $|P| \times 1$, respectively.

As in many standard books (e.g., see [7]), Petri net is a particular kind of directed graph, together with an initial marking $\mu^{0}$. The underlying graph of a Petri net is a directed, weighted, bipartite graph consisting of two kinds of nodes, called places and transitions, where arcs are either from a place to a transition or from a transition to a place. Hence, Petri nets have a well known graphical representation in which transitions are represented as boxes and places as circles with directed arcs 
interconnecting places and transitions to represent the flow relation. The initial marking is represented by placing a token in the circle representing a place $p_{i}$ as a black dot whenever $\mu^{0}\left(p_{i}\right)=1, \quad 1 \leq i \leq n=|P|$. In general, a marking $\mu$ is a mapping $\mu: P \longrightarrow \mathbb{N}$. A marking $\mu$ can hence be represented as a vector $\mu \in \mathbb{N}^{n}, n=|P|$, such that the $i^{t h}$ component of $\mu$ is the value $\mu\left(p_{i}\right)$.

In a Petri net $N$ a transition $t \in T$ is said to be enabled at $\mu$ if and only if $I^{-}(p, t) \leq \mu(p), \forall p \in P$. An enabled transition may or may not 'fire' (depending on whether the event actually takes place or not). After firing at $\mu$, the new marking $\mu^{\prime}$ is given by the rule

$$
\mu^{\prime}(p)=\mu(p)-I^{-}(p, t)+I^{+}(p, t), \text { forall } p \in P .
$$

and write $\mu \stackrel{t}{\longrightarrow} \mu^{\prime}$, whence $\mu^{\prime}$ is said to be directly reachable from $\mu$. Hence, it is clear, what is meant by a sequence like

$$
\mu^{0} \stackrel{t_{1}}{\longrightarrow} \mu^{1} \stackrel{t_{2}}{\longrightarrow} \mu^{2} \stackrel{t_{3}}{\longrightarrow} \mu^{3} \cdots \stackrel{t_{k}}{\longrightarrow} \mu^{k},
$$

which simply represents the fact that the transitions

$$
t_{1}, t_{2}, t_{3}, \ldots, t_{k}
$$

have been successively fired to transform the marking $\mu^{0}$ into the marking $\mu^{k}$. The whole of this sequence of transformations is also written in short as $\mu^{0} \stackrel{\sigma}{\longrightarrow} \mu^{k}$, where $\sigma=t_{1}, t_{2}, t_{3}, \ldots, t_{k}$.

A marking $\mu$ is said to be reachable from $\mu^{0}$, if there exists a sequence of transitions which can be successively fired to obtain $\mu$ from $\mu^{0}$. The set of all markings of a Petri net $N$ reachable from a given marking $\mu$ is denoted by $\mathcal{M}(N, \mu)$ and, together with the arcs of the form $\mu^{i} \stackrel{t_{r}}{\longrightarrow} \mu^{j}$, represents what in standard terminology called the reachability graph $R(N, \mu)$ of the Petri net $N$. If the reachability graph has no cycle then it is called reachability tree.

A place in a Petri net is safe if the number of tokens in that place never exceeds one. A Petri net is safe if all its places are safe.

The preset of a transition $t$ is the set of all input places to $t$, i.e., ${ }^{\bullet} t=\left\{p \in P: I^{-}(p, t)>0\right\}$. The postset of $t$ is the set of all output places from $t$, i.e., $t^{\bullet}=\left\{p \in P: I^{+}(p, t)>0\right\}$. Similarly, $p^{\prime} s$ preset and postset are ${ }^{\bullet} p=\left\{t \in T: I^{+}(p, t)>0\right\}$ and $p^{\bullet}=\left\{t \in T: I^{-}(p, t)>0\right\}$, respectively.

A pair of a place $p$ and a transition $t$ is called a self-loop if $p$ is both an input and output place of $t$ (see [8]).

Let $N=\left(P, T, I^{-}, I^{+}, \mu^{0}\right)$ be a Petri net with $|P|=n$ and $|T|=m$, the incidence matrix $I=\left[a_{i j}\right]$ is an $n \times m$ matrix of integers and its entries are given by $a_{i j}=a_{i j}^{+}-a_{i j}^{-}$where $a_{i j}^{+}=I^{+}\left(p_{i}, t_{j}\right)$ is the number of arcs from transition $t_{j}$ to its output place $p_{i}$ and $a_{i j}^{-}=I^{-}\left(p_{i}, t_{j}\right)$ is the number of arcs from place $p_{i}$ to its output transition $t_{j}$, i.e., in other words, $I=I^{+}{ }_{-} I^{-}$.

\section{MAIN RESULTS}

In order to establish the main result of this paper, The following definitions are needed

DEFINITION 1. [9] Let $N=\left(P, T, I^{-}, I^{+}, \mu^{0}\right)$ be a Petri net and $Z$ be a subnet of $N$. Then $Z$ is called a strong chain cycle (SCC) of $N$ or $N$ is said to have a strong chain cycle (SCC) $Z$, if $|\bullet t|=2,\left|p^{\bullet}\right|=2$ and $\left|t^{\bullet}\right|=1 \forall p, t \in Z$. If an SCC $Z$ contains all the places of $N$ then $N$ is said to have a strong chain cycle covering all the places (see Figure 1 for illustration). Note that an SCC containing $k$ places, where $k \leq n=|P|$ will always have $k$ self-loops.

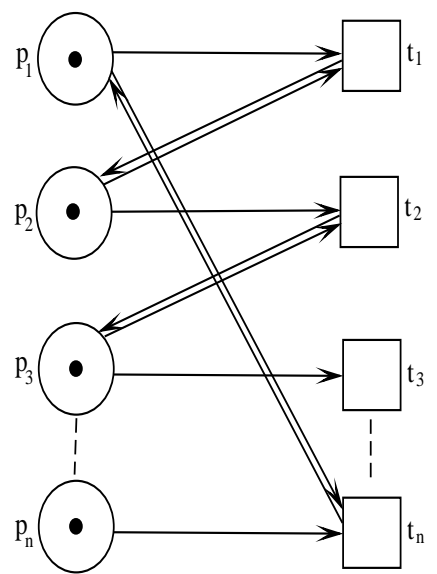

Fig. 1. Strong chain cycle

Definition 2. Let $N_{n, m}$ be a Petri net with $n$ places and $m$ transitions, having the following properties (i) $n \leq m$, (ii) the $i^{\text {th }}$ transition $t_{i}$ is contained in exactly $n-i$ self-loops, $i=1,2, \cdots, n$ and (iii) the sub-Petri net $N_{n, n}^{*}$ induced by the places $p_{1}, p_{2}, \cdots, p_{n}$ and transitions $t_{1}, t_{2}, \cdots, t_{n}$ forming identity matrix $I_{n}$ in the incidence matrix $I=I^{+}-I^{-}=I[i, j]=$ 1 , if $i=j$ and 0 otherwise, for $1 \leq i \leq n$ and $i \leq j \leq n$, of $N_{n, m}$ does not contain

\section{as a sub-Petri net.}

If the Petri net $N_{n, m}$ is 1-safe then the structure shown in Figure 2 will not exist because on firing of $t_{j}, j=1,2, \cdots, m$ Petri net will not remain safe.

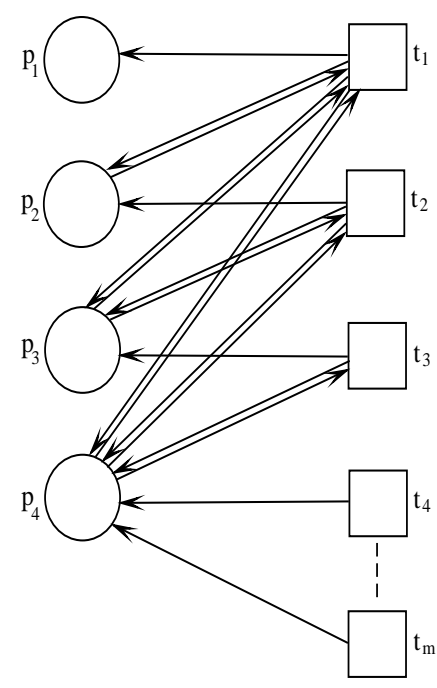

Fig. 2. A non-safe Petri net

Lemma 3. If a 1-safe Petri net $N=\left(P, T, I^{-}, I^{+}, \mu^{0}\right)$, $|P|=|T|=n$ having $I_{n}$ as its incidence matrix, containing an SCC between two places and two transitions with $\mu^{0}(p)=1$, $\forall p \in P$, then $N$ is not a Boolean Petri net. 
Proof. Suppose $N=\left(P, T, I^{-}, I^{+}, \mu^{0}\right)$ has an SCC between any two places $p_{i}, p_{j}$ and two transitions $t_{i}, t_{j}$. This means that $\left(p_{i}, t_{j}\right)$ is a single arc in $N$ if $i=j$ and symmetric arc in $N$ if $i \neq j$. Since $\mu^{0}(p)=1, \forall p \in P$, all the transitions are enabled and fire. After firing of the transitions $t_{i}$ and $t_{j}$ in the first stage, we will get the ' 0 ' at the $i^{\text {th }}$ and $j^{\text {th }}$ places respectively. After firing, these transitions become dead because $\left|{ }^{\bullet} t_{i}\right|=\left|{ }^{\bullet} t_{j}\right|=2$ as $t_{i}$ and $t_{j}$ lie on SCC. Therefore in the next stage of firing, we cannot get the marking vector whose $i^{\text {th }}$ as well as $j^{\text {th }}$ components are zero simultaneously. Therefore, $N$ does not generate all $2^{n}$ binary $n$-vectors as its marking vectors and hence is not Boolean.

Lemma 4. [9] If a 1-safe Petri net $N=\left(P, T, I^{-}, I^{+}, \mu^{0}\right)$, $|P|=n$, is Boolean then $|P| \leq|T|$.

LEMMA 5. [10] A disconnected Petri net having $n$ components of $K_{2} \cong \bigodot \longrightarrow \square$ is Boolean.

In the following theorem, which is the main result of this paper, we give the construction of a minimum crisp Boolean Petri net. By a minimum crisp Boolean Petri net we mean a Petri net which is Boolean and generates all the marking vectors exactly once using the minimum number of transitions.

Theorem 6. A 1-safe Petri net $N_{n, n}:=N_{n, n}^{*}$ with $\mu^{0}(p)=1, \forall p \in P$, is a minimum crisp Boolean Petri net.

PROOF. The theorem is proved by the Principle of Mathematical Induction (PMI) on $|P|=n$. Let $\mathrm{n}=1$. Then $N_{1,1}^{*}$ is a Petri net with one place $p_{1}$ and one transition $t_{1}$. This means $N_{1,1}^{*}$ has no self-loop as shown in Figure 3. Further, since $\mu^{0}\left(p_{1}\right)=1$, it is easy to verify that the reachability tree $R\left(N_{1,1}^{*}, \mu^{0}\right)$ of $N_{1,1}^{*}$ as shown in Figure 4 contains all the binary 1-vectors, namely (1), (0) exactly once and after firing of the transition $t_{1}$, it becomes dead.

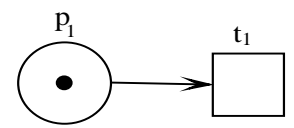

Fig. 3. $N_{1,1}^{*}$

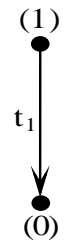

Fig. 4. $R\left(N_{1,1}^{*}, \mu^{0}\right)$

Next, let $n=2$. Then $N_{2,2}^{*}$ is a Petri net with two places namely, $p_{1}, p_{2}$ and two transitions namely, $t_{1}, t_{2}$. The structure of $N_{2,2}^{*}$ is shown in Figure 5. Since $\mu^{0}(p)=1, \forall p \in P$, both the transitions $t_{1}, t_{2}$ are enabled and fire. After firing of $t_{1}$ and $t_{2}$ in the first stage, we get marking vectors $(0,1)$ and $(1,0)$ respectively. In the second stage of firing, at the marking vector $(0,1)$, only transition $t_{2}$ is enabled and gives the marking vector $(0,0)$ after firing and at this marking vector all the transitions become dead. On the other hand, at the marking vector $(1,0)$, no transition is enabled. Hence. it is clear that the reachability tree $R\left(N_{2,2}^{*}, \mu^{0}\right)$ of $N_{2,2}^{*}$ as shown in Figure 6 contains all the $4=2^{2}$, binary

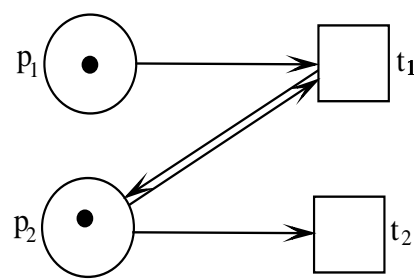

Fig. 5. $\quad N_{2,2}^{*}$

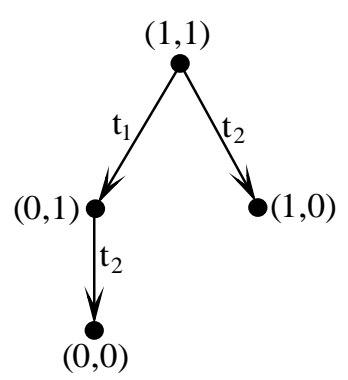

Fig. 6. $R\left(N_{2,2}^{*}, \mu^{0}\right)$

2 -vectors $\left(a_{1}, a_{2}\right), a_{1}, a_{2} \in\{0,1\}$ exactly once having the minimum number of transitions.

We can construct $N_{2,2}^{*}$ from $N_{1,1}^{*}$ and $R\left(N_{2,2}^{*}, \mu^{0}\right)$ from $R\left(N_{1,1}^{*}, \mu^{0}\right)$ procedurally as follows:

Construction of $N_{2,2}^{*}$ from $N_{1,1}^{*}$

Step 1: Take one copy of $N_{1,1}^{*}$ and one copy of $K_{2} \cong \bigodot \longrightarrow \square$, letting the place and transition in $K_{2}$ as $p_{2}$ and $t_{2}$ respectively.

Step 2: Draw the self-loop from the place $p_{2}$ of $K_{2}$ to the transition $t_{1}$ in $N_{1,1}^{*}$. In this way, we obtain the resulting structure $N_{2,2}^{\sharp}$ having two places $p_{1}, p_{2}$ and two transitions $t_{1}, t_{2}$, in which transition $t_{1}$ is contained in only one self-loop and $t_{2}$ is not contained in any self-loop as shown in Figure 7.

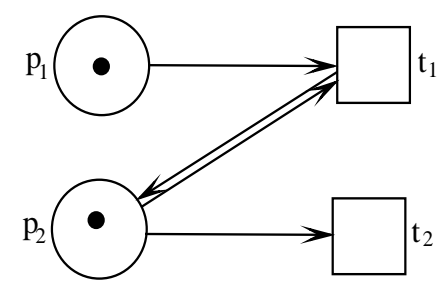

Fig. 7. $N_{2,2}^{\sharp}=: N_{2,2}^{*}$

Construction of $R\left(N_{2,2}^{*}, \mu^{0}\right)$ from $R\left(N_{1,1}^{*}, \mu^{0}\right)$

Step 1: Take two copies of $R\left(N_{1,1}^{*}, \mu^{0}\right)$. In the first copy, augment ' 0 ' at the first position of each vector of $R\left(N_{1,1}^{*}, \mu^{0}\right)$ and denote the resulting labeled tree as $R_{0}\left(N_{1,1}^{*}, \mu^{0}\right)$ as shown in Figure 8. Similarly, in the second copy, augment ' 1 ' at the first position of each vector of $R\left(N_{1,1}^{*}, \mu^{0}\right)$ and denote the resulting labeled tree as $R_{1}\left(N_{1,1}^{*}, \mu^{0}\right)$ as shown in Figure 9.

Step 2: Join the root node $(1,1)$ of $R_{1}\left(N_{1,1}^{*}, \mu^{0}\right)$ to the root node $(0,1)$ of $R_{0}\left(N_{1,1}^{*}, \mu^{0}\right)$ by an arc from $(1,1)$ to $(0,1)$ and label it as $t_{1}$ (see Figure 10). 


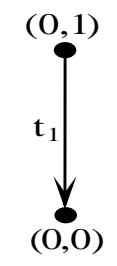

Fig. 8. $\quad R_{0}\left(N_{1,1}^{*}, \mu^{0}\right)$

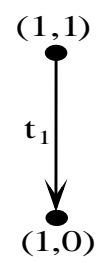

Fig. 9. $R_{1}\left(N_{1,1}^{*}, \mu^{0}\right)$

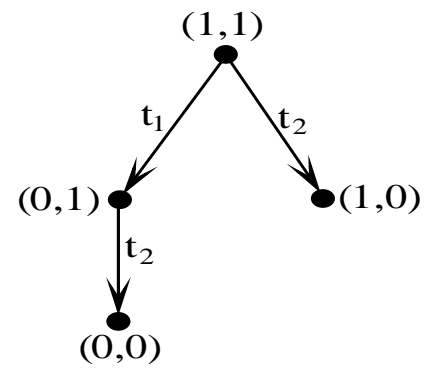

Fig. 10. $R^{*}\left(N_{2,2}^{*}, \mu^{0}\right)$

Step 3: Label the arcs of $R_{1}\left(N_{1,1}^{*}, \mu^{0}\right)$ and $R_{0}\left(N_{1,1}^{*}, \mu^{0}\right)$ as $t_{2}$ in $R^{*}\left(N_{2,2}^{*}, \mu^{0}\right)$. Thus, we get the resulting labeled tree $R^{*}\left(N_{2,2}^{*}, \mu^{0}\right)$ as shown in Figure 10 .

It is easy to see that all the vectors in $R^{*}\left(N_{2,2}^{*}, \mu^{0}\right)$ are distinct since these are obtained by fusing the nodes of $R_{1}\left(N_{1,1}^{*}, \mu^{0}\right)$ and $R_{0}\left(N_{1.1}^{*}, \mu^{0}\right)$ and are $4=2^{2}$ in number. It is clear that these are the marking vectors of $N_{2,2}^{*}$. Thus, the resulting labeled tree $R^{*}\left(N_{2,2}^{*}, \mu^{0}\right)$ obtained is indeed the reachability tree of $N_{2,2}^{*}$ because $R^{*}\left(N_{2,2}^{*}, \mu^{0}\right)$ has the same number of distinct nodes together with the same number of arcs having the same labelings (see Figure 10). By the construction of $N_{2,2}^{\sharp}$ from $N_{1,1}^{*}$ as given in Step 2, $N_{2,2}^{\sharp}=: N_{2,2}^{*}$ implies the uniqueness of its reachability tree. Hence, $R^{*}\left(N_{2,2}^{*}, \mu^{0}\right)=: R\left(N_{2,2}^{*}, \mu^{0}\right)$.

Next, let $n=3$. Then $N_{3,3}^{*}$ is a Petri net with three places namely, $p_{1}, p_{2}$ and $p_{3}$ and three transitions namely, $t_{1}, t_{2}$ and $t_{3}$. The structure of $N_{3,3}^{*}$ is shown in Figure 11. Since $\mu^{0}(p)=1$, $\forall p \in P$, all the transitions $t_{1}, t_{2}$ and $t_{3}$ are enabled and fire. After firing of $t_{1}, t_{2}$ and $t_{3}$ in the first stage, we get marking vectors $(0,1,1),(1,0,1)$ and $(1,1,0)$ respectively. In the second stage of firing, at the marking vector $(0,1,1)$, only transition $t_{2}$ and $t_{3}$ are enabled and fire giving the marking vector $(0,0,1)$ and $(0,1,0)$ respectively. On the other hand, at the marking vector $(1,0,1)$, only transition $t_{3}$ is enabled and after firing gives the marking vector $(1,0,0)$. Similarly, at the marking vector $(1,1,0)$ all the transitions become dead. In the third stage of firing, only at the marking vector $(0,0,1)$, the transition $t_{3}$ is enabled and fires giving the marking vector $(0,0,0)$. Hence. it is clear that the reachability tree $R\left(N_{3,3}^{*}, \mu^{0}\right)$ of $N_{3,3}^{*}$ (see Figure 12) contains all the $8=2^{3}$, binary 3 -vectors $\left(a_{1}, a_{2}, a_{3}\right)$, $a_{1}, a_{2}, a_{3} \in\{0,1\}$ exactly once with the minimum number of transitions.

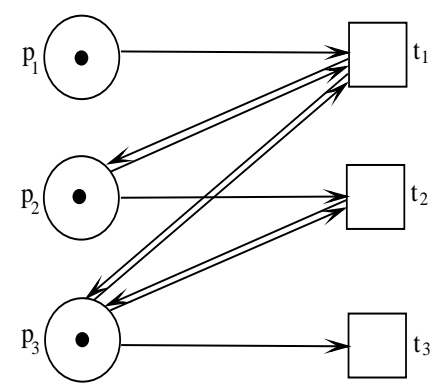

Fig. 11. $N_{3,3}^{*}$

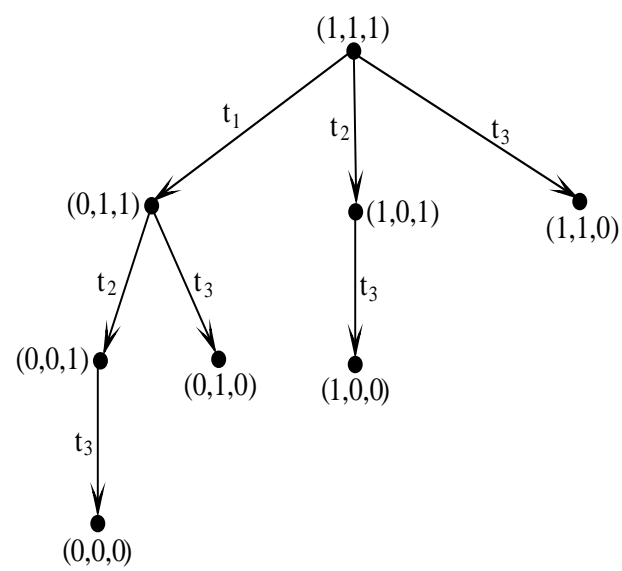

Fig. 12. $R\left(N_{3,3}^{*}, \mu^{0}\right)$

We can construct $N_{3,3}^{*}$ from $N_{2,2}^{*}$ and $R\left(N_{3,3}^{*}, \mu^{0}\right)$ from $R\left(N_{2,2}^{*}, \mu^{0}\right)$ procedurally as follows:

Construction of $N_{3,3}^{*}$ from $N_{2,2}^{*}$

Step 1: Take one copy of $N_{2,2}^{*}$ and one copy of $K_{2} \cong \bigodot \longrightarrow \square$, letting the place and transition in $K_{2}$ as $p_{3}$ and $t_{3}$ respectively. Step 2: Draw the self-loop from the place $p_{3}$ of $K_{2}$ to each $t_{i}$, $i=1,2$. In this way, we obtain the resulting structure $N_{3,3}^{\sharp}$ having three places $p_{1}, p_{2}, p_{3}$ and three transitions $t_{1}, t_{2}, t_{3}$ in which transition $t_{i}$ is contained in $(n-i)$ self-loops, $\forall i=1,2,3$, as shown in Figure 13. Thus, $N_{3,3}^{\sharp}=: N_{3,3}^{*}$.

Construction of $R\left(N_{3,3}^{*}, \mu^{0}\right)$ from $R\left(N_{2,2}^{*}, \mu^{0}\right)$

Step 1: Take two copies of $R\left(N_{2,2}^{*}, \mu^{0}\right)$. In the first copy, augment ' 0 ' at the first position of each vector of $R\left(N_{2,2}^{*}, \mu^{0}\right)$ and denote the resulting labeled tree as $R_{0}\left(N_{2,2}^{*}, \mu^{0}\right)$ as shown in Figure 14. Similarly, in the second copy, augment ' 1 ' at the first position of each vector of $R\left(N_{2,2}^{*}, \mu^{0}\right)$ and denote the resulting labeled tree as $R_{1}\left(N_{2,2}^{*}, \mu^{0}\right)$ as shown in Figure 15 .

Step 2: Join the root node $(1,1,1)$ of $R_{1}\left(N_{2,2}^{*}, \mu^{0}\right)$ to the root node $(0,1,1)$ of $R_{0}\left(N_{2,2}^{*}, \mu^{0}\right)$ by an arc from $(1,1,1)$ to $(0,1,1)$ and label it as $t_{1}$ (see Figure 16). 


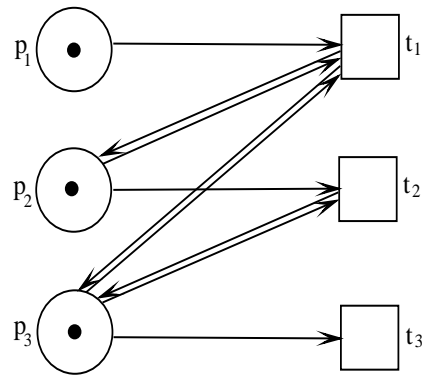

Fig. 13. $\quad N_{3,3}^{\sharp}=$ : $N_{3,3}^{*}$

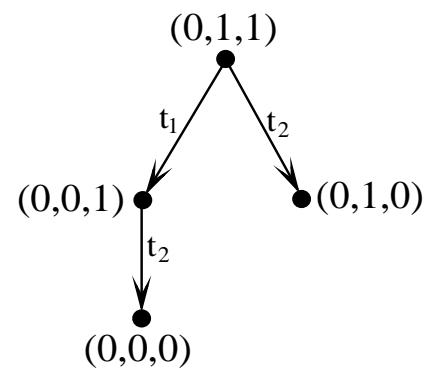

Fig. 14. $R_{0}\left(N_{2,2}^{*}, \mu^{0}\right)$

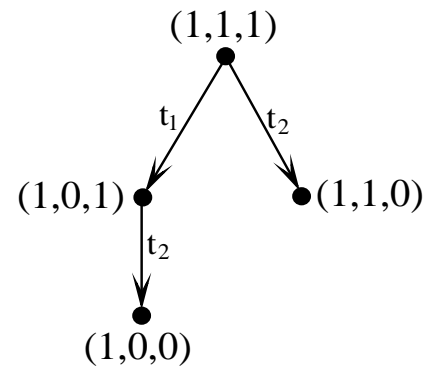

Fig. 15. $R_{1}\left(N_{2,2}^{*}, \mu^{0}\right)$

Step 3: Increase by one the suffixes of labeled arcs of $R_{1}\left(N_{2,2}^{*}, \mu^{0}\right)$ and $R_{0}\left(N_{2,2}^{*}, \mu^{0}\right)$ except the labeled arc $t_{1}$ as in Step 2. Thus, we get the resulting labeled tree $R^{*}\left(N_{3,3}^{*}, \mu^{0}\right)$ (see Figure 16)

In fact, all the vectors in $R^{*}\left(N_{3,3}^{*}, \mu^{0}\right)$ are distinct since these are obtained by fusing of the nodes of $R_{1}\left(N_{2,2}^{*}, \mu^{0}\right)$ and $R_{0}\left(N_{2,2}^{*}, \mu^{0}\right)$ and they are $2^{2}+2^{2}=2^{3}$ in number. Also, labeling of arcs in the resulting tree $R^{*}\left(N_{3,3}^{*}, \mu^{0}\right)$ is the same as $R\left(N_{3,3}^{*}, \mu^{0}\right)$. Thus, the resulting tree $R^{*}\left(N_{3,3}^{*}, \mu^{0}\right)$ obtained is indeed the reachability tree of $N_{3,3}^{*}$ because $R^{*}\left(N_{3,3}^{*}, \mu^{0}\right)$ has the same number of distinct nodes together with the same number of arcs having the same labelings. By the construction as given in Step 2 of $N_{3,3}^{\sharp}$ from $N_{3,3}^{*}, N_{3,3}^{\sharp}$ $=: N_{3,3}^{*}$ implies the uniqueness of its reachability tree. Hence, $R^{*}\left(N_{3,3}^{*}, \mu^{0}\right)=: R\left(N_{3,3}^{*}, \mu^{0}\right)$.

Now, assume that the result is true for $n=k$ places. That means, we have a crisp Boolean Petri net $N_{k, k}^{*}$ with $k$ places namely, $p_{1}, p_{2}, \cdots, p_{k}$ and $k$ transitions namely, $t_{1}, t_{2} \cdots, t_{k}$, where the transition $t_{i}$ is contained in $(k-i)$ self-loops, $\forall i=1,2, \cdots, k$ (see Figure 17).

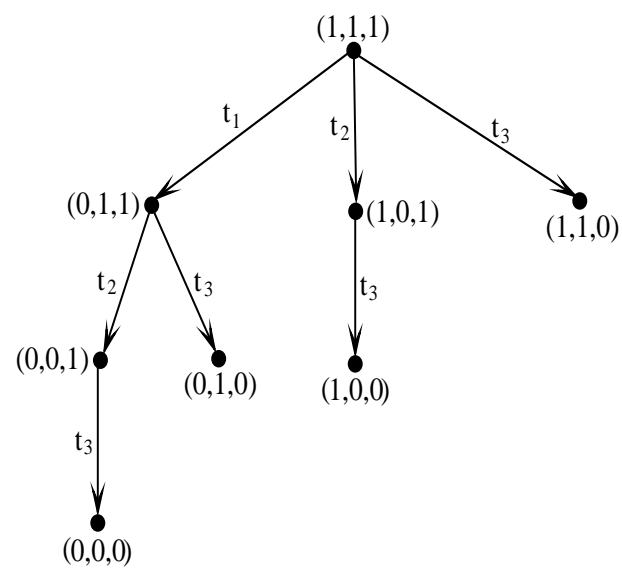

Fig. 16. $R^{*}\left(N_{3,3}^{*}, \mu^{0}\right)=: R\left(N_{3,3}^{*}, \mu^{0}\right)$

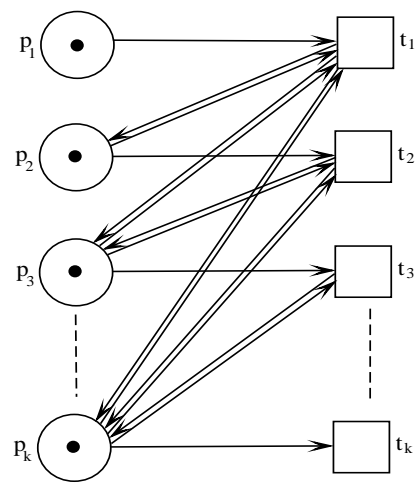

Fig. 17. $N_{k, k}^{*}$

Now, show that the result is true for $n=k+1$. For this purpose, construct $N_{k+1, k+1}^{*}$ from $N_{k, k}^{*}$ and $R\left(N_{k+1, k+1}^{*}, \mu^{0}\right)$ from $R\left(N_{k, k}^{*}, \mu^{0}\right)$ procedurally as follows:

Construction of $N_{k+1, k+1}^{*}$ from $N_{k, k}^{*}$

Step I: Take one copy of $N_{k, k}^{*}$ and one copy of $K_{2} \cong \bigodot \longrightarrow \square$, letting the place and transition of $K_{2}$ denoted as $p_{k+1}$ and $t_{k+1}$ respectively.

Step II: Draw a self-loop between $p_{k+1}$ to each $t_{i}, i=$ $1,2, \cdots, k$. So, we obtain the resulting structure $N_{k+1, k+1}^{\sharp}$ for $k+1$ places namely, $p_{1}, p_{2}, \cdots, p_{k+1}$ and $k+1$ transitions namely, $t_{1}, t_{2}, \cdots, t_{k+1}$ in which the transition $t_{i}$ is contained in $(k+1)-i$ self-loops, $\forall i=1,2, \cdots,(k+1)$, as shown in Figure 18. Thus, $N_{k+1, k+1}^{\sharp}=: N_{k+1, k+1}^{*}$.

Construction of $R\left(N_{k+1, k+1}^{*}, \mu^{0}\right)$ from $R\left(N_{k, k}^{*}, \mu^{0}\right)$

Step I: Take two copies of $R\left(N_{k, k}^{*}, \mu^{0}\right)$. In the first copy, augment ' 0 ' at the first position of each vector of $R\left(N_{k, k}^{*}, \mu^{0}\right)$ and denote the resulting labeled tree as $R_{0}\left(N_{k, k}^{*}, \mu^{0}\right)$. Similarly, in the second copy, augment ' 1 ' at the first position of each vector of $R\left(N_{k, k}^{*}, \mu^{0}\right)$ and denote the resulting labeled tree as $R_{1}\left(N_{k, k}^{*}, \mu^{0}\right)$. Hence, all the augmented vectors in $R_{0}\left(N_{k, k}^{*}, \mu^{0}\right)$ and $R_{1}\left(N_{k, k}^{*}, \mu^{0}\right)$ are distinct.

Step II: Join the root node $(1,1,1, \cdots, 1)$ of $R_{1}\left(N_{k, k}^{*}, \mu^{0}\right)$ to the root node $(0,1,1, \cdots, 1)$ of $R_{0}\left(N_{k, k}^{*}, \mu^{0}\right)$ by an arc from $(1,1, \cdots, 1)$ to $(0,1, \cdots, 1)$ and label it as $t_{1}$. 


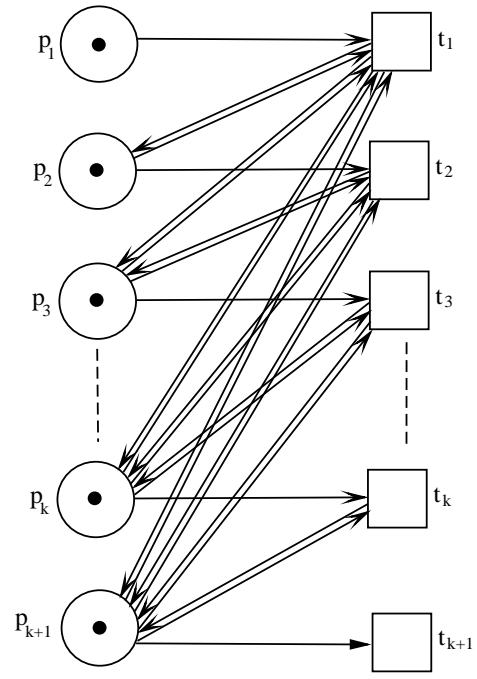

Fig. 18. $\quad N_{k+1, k+1}^{\sharp}=$ : $N_{k+1, k+1}^{*}$

Step III: Increase by one the suffixes of labeled arcs of $R_{1}\left(N_{k, k}^{*}, \mu^{0}\right)$ and $R_{0}\left(N_{k, k}^{*}, \mu^{0}\right)$ except the labeled arc $t_{1}$ as in Step II. Thus, we get the resulting labeled tree $R^{*}\left(N_{k+1, k+1}^{*}, \mu^{0}\right)$.

In fact, all the vectors in $R^{*}\left(N_{k+1, k+1}^{*}, \mu^{0}\right)$ are distinct since these are obtained by fusing the nodes of $R_{1}\left(N_{k, k}^{*}, \mu^{0}\right)$ and $R_{0}\left(N_{k, k}^{*}, \mu^{0}\right)$ and they are $2^{k}+2^{k}=2^{k+1}$ in number. Also, labeling of arcs in the resulting tree $R^{*}\left(N_{k+1, k+1}^{*}, \mu^{0}\right)$ is the same as in $R\left(N_{k+1, k+1}^{*}, \mu^{0}\right)$. Thus, the resulting tree $R^{*}\left(N_{k+1, k+1}^{*}, \mu^{0}\right)$ obtained is indeed the reachability tree of $N_{k+1, k+1}^{*}$ because $R^{*}\left(N_{k+1, k+1}^{*}, \mu^{0}\right)$ has the same number of distinct nodes together with the same number of arcs having the same labelings. By the construction as given in Step II of $N_{k+1, k+1}^{\sharp}$ from $N_{k+1, k+1}^{*}, N_{k+1, k+1}^{\sharp}=$ : $N_{k+1, k+1}^{*}$ implies the uniqueness of its reachability tree. Hence, $R^{*}\left(N_{k+1, k+1}^{*}, \mu^{0}\right)=: R\left(N_{k+1, k+1}^{*}, \mu^{0}\right)$.

Hence, the result follows by PMI.

The 1-safe Petri net $N_{n, m}$ where $n \neq m$ with $\mu^{0}(p)=1, \forall p \in$ $P$ also generates all the binary $n$-vectors. In this case repetitions may occur. That is $N_{n, m}$ is a Boolean Petri net but not necessarily crisp. In any 1-safe Petri net $N=\left(P, T, I^{-}, I^{+}, \mu^{0}\right)$, $|P|=|T|=n$, with $\mu^{0}(p)=1, \forall p \in P$, at any stage in the dynamics of $N$ when binary $n$-vectors at Hamming distance $k$ from $\mu^{0}$ are being generated all the binary $n$-vectors of Hamming distance less than $k$ have already been generated.

Now, the uniqueness of 1-safe Petri net $N_{n, n}=: N_{n, n}^{*}$ which is crisp Boolean will be proved in the next theorem.

Theorem 7. The 1-safe Petri net $N_{n, n}:=N_{n, n}^{*}$ is the unique minimum crisp Boolean Petri net.

Proof. The existence of the crisp Boolean Petri net $N_{n, n}^{*}$ has been already proved in Theorem 1 . Here, the uniqueness of $N_{n, n}^{*}$ will be established. Since $N_{n, n}^{*}$ has $n$ places and $n$ transitions, by Lemma $2, N_{n, n}^{*}$ is minimum. Let $N$ be any minimum crisp Boolean Petri net. We claim, $N \cong N_{n, n}^{*}$. Since $N$ is crisp, $|P|=n$ and minimality of $N$ implies that $|T|=n$. Furthermore, $\mu^{0}(p)=1, \forall p \in P$. For generating all the ${ }^{n} C_{1}$ binary $n$-vectors as marking vectors of Hamming distance 1 from $\mu^{0}$, we must have a spanning subgraph $N^{\prime}$ of $N$ consisting of $n$ copies of $K_{2} \cong \odot \longrightarrow \square$, this leads to a contradiction about crisp Boolean
Petri net (by Lemma 3). Suppose in $N$, there is an arc from $t_{i}$ to $p_{j}$ and not from $p_{j}$ to $t_{i}$, for $i \neq j$. Then at the first stage of firing at $\mu^{0}$, the safeness of $N$ is violated. Therefore, there is no $\operatorname{arc}$ in $N$ from $t_{i}$ to $p_{j}$, for $i \neq j$. Hence, whenever there is an arc from $t_{i}$ to $p_{j}$, for $i \neq j$, there exists an arc from $p_{j}$ to $t_{i}$ so that they form a self-loop. Next, suppose in $N$, there is an arc from $p_{j}$ to $t_{i}$ and not from $t_{i}$ to $p_{j}$, for $i \neq j$. Then in the first stage of firing of $t_{i}$ we will get at least one marking vector of Hamming distance 2 from $\mu^{0}$ whose $i^{t h}$ and $j^{t h}$ components are zero and would not get the marking vector of Hamming distance 1 whose $i^{\text {th }}$ component is zero and $j^{\text {th }}$ component is 1 or vice-versa. $|T|=n$ implies that in the next stage of firing, the marking vector of Hamming distance 1 from $\mu^{0}$ cannot get generated. By Remark 3 it implies that when $k=2$, we get the contradiction to our assumption that $N$ is crisp. Therefore, whenever, there is an arc from $p_{j}$ to $t_{i}, i \neq j$, there exists an arc from $t_{i}$ to $p_{j}$ also; in other words, $p_{i}$ and $t_{j}$ form a self-loop, for $i \neq j$. These arguments imply either $p_{i}$ and $t_{j}$ are not adjacent or they form a self-loop, for $i \neq j$. If for all $i \neq j ; p_{i}$ and $t_{j}$ are not adjacent then $N=n \odot \longrightarrow \square$ which is not crisp Boolean (see Lemma 3). Therefore, there do exist self-loops $\left(p_{i}, t_{j}\right)$ for some $i \neq j$. If $n=1, N \cong N_{1,1}^{*}$ and we are through. Hence, suppose $n \geq 2$. Since there is at least one self-loop in $N, N$ has less connected components than those in $N^{\prime}$. The following cases are under consideration, namely:

Case 1: $N^{\prime}$ contains $C_{1} \cong \stackrel{p_{i}}{\longrightarrow} \longrightarrow \stackrel{t_{i}}{\square}$ as a component. Since $n \geq$ 2 there exists a component $C_{2}$ of $N^{\prime}$. Now two subcases arise.

Subcase 1: $C_{2} \cong \stackrel{p_{r}}{\longrightarrow} \longrightarrow \stackrel{t_{r}}{\square}$ i.e., $C_{2}$ has only one component of $N^{\prime}$. If there is no self-loop in $N$ connecting $C_{1}$ and $C_{2}$ in $N^{\prime}$ then a marking vector of Hamming distance 2 from $\mu^{0}$ is repeated, a contradiction to our assumption. Therefore, there must be a selfloop connecting $C_{1}$ and $C_{2}$ in $N$. Without loss of generality, let $C_{1}=\left(p_{i}, t_{i}\right), C_{2}=\left(p_{r}, t_{r}\right)$ and self-loop $\left(p_{i}, t_{r}\right)$ or $\left(p_{r}, t_{i}\right)$. If both self-loop $\left(p_{i}, t_{r}\right)$ and $\left(p_{r}, t_{i}\right)$ exist then by Lemma $1, N$ is not crisp Boolean. Therefore, only one of these self-loops exists. Without loss of generality, assume that $\left(p_{r}, t_{i}\right)$ is a self-loop that connects $C_{1}$ and $C_{2}$. Thus for $n=2, N \cong N_{2,2}^{*}$.

Subcase 2: $C_{2}$ has at least two components of $N^{\prime}$. Let these components of $N^{\prime}$ be $\stackrel{p_{r}}{\bigodot} \longrightarrow \stackrel{t_{r}}{\square}$ and $\stackrel{p_{s}}{\bigodot} \longrightarrow \stackrel{t_{s}}{\longrightarrow}$. Since $C_{2}$ is connected, there are at least $\frac{\left|C_{2}\right|}{2}-1$ self-loops in $C_{2}$. If $\left|C_{2}\right|=4$ then in $C_{2}$ there are at least $\frac{4}{2}-1=1$ selfloop. Now, without loss of generality, suppose $\left(p_{s}, t_{r}\right)$ is a self-loop. After firing in $N$, we get all the binary vectors of Hamming distance 1 from $\mu^{0}$ without repetitions. We get the binary vectors $\left(a_{1}, a_{2}, \cdots, a_{i}, \cdots, a_{r}, a_{r+1} \cdots, a_{s}, \cdots, a_{n}\right)$ where $a_{i}=0, a_{j}=1, \forall j \neq i, i<r<s$ and $\left(a_{1}, a_{2}, \cdots, a_{i}, \cdots, a_{r}, a_{r+1}, \cdots, a_{s}\right.$,

$\left.\cdots, a_{n}\right)$ where $a_{r}=0, a_{k}=1, \forall k \neq r$. Now, consider the marking vector $\left(a_{1}, a_{2}, \cdots, a_{i-1}, 0, a_{i+1} \cdots, a_{r}, a_{s}, \cdots, a_{n}\right)$, where $a_{j}=1, \forall j \neq i$. At this marking, $t_{r}$ is enabled and fires, whence we get $\left(a_{1}, a_{2}, \cdots, a_{i-1}, 0, a_{i+1}, \cdots, a_{r-1}\right.$

$\left., 0, a_{r+1}, \cdots, a_{n}\right)$, where $a_{j}=1, \forall j \neq i, r$. Similarly, at the marking vector $\left(a_{1}, a_{2}, \cdots, a_{i}, \cdots, a_{r-1}, 0, a_{r+1}, \cdots, a_{n}\right)$, where $a_{r}=0, a_{j}=1, \forall j \neq r$, the transition $t_{i}$ is enabled and after firing gives the marking vector $\left(a_{1}, a_{2}, \cdots, a_{i-1}\right.$,

$\left.0, a_{i+1} \cdots, a_{r-1}, 0, a_{r+1}, \cdots, a_{n}\right)$, where $a_{j}=1, \forall j \neq i, r$ which is obtained again, a contradiction to our initial assumption about $N$. The same arguments hold when $\left|C_{2}\right|>4$, i.e., $C_{2}$ has more than 2 components of $N^{\prime}$.

Case 2: The above arguments imply that every component of $N$ has at least two components of $N^{\prime}$. But, then the argument given in Subcase 2 above leads us to the same contradiction.

It follows that $N$ is connected. Therefore, $N$ should have at least $\frac{|N|}{2}-1$ self-loops. Since $|P|=|T|=n, N$ cannot contain a strong chain cycle (SCC) by virtue of Lemma 1 . We claim, there exists a transition in $N$ which is joined by self-loops to all but 
one place. Suppose there exists no transition joined by self-loops to all but one of the places in $N$. This implies, there is a transition say $t_{1}$ not joined by self-loops to at least two places, namely to $p_{1}$ and one other place say $p_{2}$. Since, $N$ generates all the binary $n$-vectors as marking vectors, in particular $\underline{a}=(0,1,1, \cdots, 1)$ and $\underline{b}=(1,0,1, \cdots, 1)$ are generated by $N$ in the first stage of firing. Now, the vector $a$ at which $t_{2}$ is enabled and fires giving rise to vector $\underline{a^{\prime}}=(0,0,1, \cdots, 1)$, is also obtained by firing $t_{1}$ at $b$, a contradiction to our assumption about $N$ that $N$ is crisp. Thus $t_{1}$ is joined to all the places $p_{i}$ by self-loops except $p_{1}$. Next, suppose there exists $t_{j}, j \neq 1$, joined by self-loops, to all but one of the places, in particular $t_{j}$ will be joined to $p_{1}$ by a self-loop. Then, there is an SCC formed by the places $p_{1}$ and $p_{j}$ and transitions $t_{1}$ and $t_{j}$ respectively. This contradicts Lemma 1. Now, we claim there exists a transition joined by self-loops to all but two of the places. Suppose this is not true, i.e., there exists a transition which is not joined by self-loops to at least three places. Without loss of generality, suppose $t_{2}$ is such a transition. Since $t_{2}$ is not joined by self-loops to $p_{1}, p_{2}$ and $p_{3}$, let $p_{j}$ be a place $j \neq 1,2,3$ to which $t_{2}$ is joined by a self-loop. Since, $N$ generates all the binary $n$-vectors as marking vectors, in particular $c=(1,1,0,1, \cdots, 1)$ and $\underline{b}=(1,0,1, \cdots, 1)$ are generated by $N$ in the first stage of firing. Now, the vector $\underline{c}$ at which $t_{2}$ is enabled and fires giving rise to vector $\underline{c}^{\prime}=(1,0,0,1, \cdots, 1)$ (as $t_{2}$ is not joined by self-loop to $p_{2}$ ) is also obtained by firing $t_{3}$ at $\underline{b}$, a contradiction to our assumption about $N$ that it generates all the binary $n$-vectors exactly once, establishing the contradiction to our assumption that $N$ is crisp Boolean. Thus $t_{2}$ is joined by self-loops to all but two of the places. These two places are $p_{1}$ and $p_{2}$ because $\left(p_{i}, t_{i}\right)$ is not a self-loop, $\forall i$ and if $\left(p_{1}, t_{2}\right)$ is a self-loop then SCC will be found which contradicts Lemma 1. Next suppose $t_{j}, j \neq 1,2$, is joined by self-loop to all but two of the places then in the structure of Petri net SCC will be formed which contradicts the Lemma 1. Continuing these arguments, in general that for each $j, 1 \leq j \leq n, t_{j}$ is joined by self-loops to exactly $n-j$ places.

Thus $N \cong N_{n, n}^{*}$ is the unique minimum crisp Boolean Petri net.

\section{CONCLUSIONS AND SCOPE}

The existence and uniqueness of minimum crisp Boolean Petri nets. A computationally good characterization of such Petri nets in general is highly desirable since the instances where we need such Petri nets for applications are imaginably (as well as arguably) large in number as pointed out in [2]. This has been a hotly pursued research problem. The general problem of characterizing such a 1-safe Petri net $N$ is still open.

\section{REFERENCES}

[1] Petri, C.A., Kommunikation mit automaten, Schriften des Institutes fur Instrumentelle Mathematik, Bonn 1962.

[2] Kansal, S., Singh, G.P. and Acharya, M., On Petri nets generating all the binary $n$-vectors, Scientiae Mathematicae Japonicae, 71(2), 2010, 209-216.

[3] Kansal, S., Singh, G.P. and Acharya, M., 1-Safe Petri nets generating every binary $n$-vector exactly once, Scientiae Mathematicae Japonicae, 74(1), 2011, 29-36.

[4] Peterson, J.L., Petri net Theory and the Modeling of Systems, Englewood Cliffs, NJ: Prentice-Hall, Inc., 1981.

[5] Harary, F., Graph theory, Addison-Wesley, Massachusettes, Reading, 1969

[6] Jensen, K., Coloured Petri nets, Lecture notes in Computer Science, Springer-Verlag, Berlin, 254, 1986, 248-299.

[7] Reisig, W., Petri nets, Springer-Verleg, New York, 1985.

[8] Murata, T., Petri nets: Properties, analysis and applications, Proc. IEEE, 77(4), 1989, 541-580.

[9] Kansal, S., Acharya, M. and Singh, G.P., Boolean Petri nets. In: Petri nets - Manufacturing and Computer Science (Ed.: Pawel Pawlewski), 381-406; Chapter 17. In-Tech Global Publisher, 2012, ISBN 978-953-51-0700-2.

[10] Singh, G.P., Some advances in the theory of Petri nets, Ph.D. Thesis, submitted to the University of Delhi, Delhi, December 5, 2012. 